\title{
Determinación del punto de carga cero y punto isoeléctrico de dos residuos agrícolas y su aplicación en la remoción de colorantes
}

\author{
Determination of the point of zero charge and \\ isoelectric point of two agricultural wastes and \\ their application in the removal of colorants
}

\section{Determinação do ponto de carga zero e ponto isoelétrico de dois resíduos agrícolas e sua aplicação na remoção de corantes.}

\author{
Fredy Alberto Amaringo Villa ${ }^{1}$ \& Angelina Hormaza Anaguano² \\ ${ }^{1}$ Químico, Especialista en Educación Ambiental, Estudiante de maestría en Ciencias, Universidad \\ Nacional de Colombia - Sede Medellín. ²Químico, Magister en Ciencias- Química Orgánica, \\ Doctor Rerum Naturualien, Doctora en Ciencias Químicas. \\ 1,2Escuela de Química, Facultad de Ciencias, Universidad Nacional de Colombia. Medellín, Colombia.
}

1faamaringov@unal.edu.co, ${ }^{2}$ ahormaza@unal.edu.co

\section{Resumen}

Los residuos agroindustriales han mostrado características adsorbentes, y con ello su posible uso alternativo para la remoción de contaminantes disueltos presentes en los efluentes industriales. La caracterización química de la superficie del material adsorbente permite establecer su capacidad para retener ciertos contaminantes iónicos. En el presente estudio se determinó el punto de carga cero (PZC) y el punto isoeléctrico (IEP) de dos residuos agrícolas, cascarilla de arroz y corteza de coco, con el fin de establecer la distribución de cargas sobre su superficie y posteriormente evaluar su capacidad de remoción de colorantes aniónicos o catiónicos disueltos. Para ello se utilizó el método de la deriva del $\mathrm{pH}$ y del potencial zeta, variando el intervalo de $\mathrm{pH}$ entre 2.0 y 11 y manteniendo constante las demás variables. Para la cascarilla de arroz se obtuvo un PZC de 5.40 y un IEP de 9.10 y para la corteza de coco un PZC de 4.61 y un IEP de 9.45, señalando una distribución de cargas predominantemente positivas sobre su superficie. La posterior evaluación de esta característica comprobó su afinidad para la remoción de colorantes iónicos. Se alcanzó una remoción del 93\% del colorante catiónico rojo básico 46 (RB46) a un pH mayor a 5.0 y una retención del $73 \%$ del colorante aniónico rojo 40 (R40) a un $\mathrm{pH}=2.0$ sobre cascarilla de arroz. La determinación del PZC y IEP de los adsorbentes posibilita el establecimiento de las mejores condiciones para la adsorción de colorantes iónicos en solución.

Palabras clave: adsorción, cascarilla de arroz, corteza de coco, rojo básico 46, rojo 40.

\section{Abstract}

Agro-industrial wastes have shown adsorptive properties and therefore can be considered as a potential alternative for the removal of dissolved contaminants present in industrial effluents. The chemical composition of the absorbate's surface allows it to stablish its capacity to retain certain ionic contaminants. This study, determinates the point of zero charge (PZC) and the isoelectric 
point (IEP) of two agricultural wastes, rice husks and coconut husk, with the end of establishing the distribution of charges on the surface and subsequently to evaluate their capacity for the removal dissolved anionic or cationic colorants. In order to accomplish this, the study utilized the methods of $\mathrm{pH}$ drift and zeta potential varying the $\mathrm{pH}$ interval between 2.0 and 11 and maintaining the other variables at a constant. A PZC of 5.40 and an IEP of 9.10 were obtained for the rice husk, and a PZC of 4.61 and an IEP of 9.45 for the coconut husk, indicating a distribution of predominately positive charge on the surface. Further study of this characteristic confirmed its affinity for the removal of ionic colorants. The rice husk showed a removal of $93 \%$ of the cationic colorant basic red 46 (RB46) with at a $\mathrm{pH}$ higher than 5.0 and a retention of $73 \%$ of the anionic red 40 colorant(R40) at $\mathrm{pH}=2.0$ on rice husks. The determination of the PZC and IEP of the adsorbents allows for the establishment of better conditions for the adsorption of ionic colorants in solution.

Keywords: adsorption, rice husks, coconut shell, basic red 46, red 40 .

\section{Resumo}

Os resíduos agroindustriais têm se mostrado com características absorventes e com ele seu possível uso alternativo para a remoção de contaminantes dissolvidos presentes nos efluentes industriais. A caracterização química da superfície do material absorvente permite estabelecer sua capacidade para reter certos contaminantes iônicos. Na pesquisa que se segue determinou-se o ponto de carga zero (PCZ) e o ponto isoelétrico (PIE) dos resíduos agrícolas, casca de arroz e de coco, com a finalidade de estabelecer a distribuição de cargas sobre a superfície e posteriormente avaliar sua capacidade de remoção de corantes aniônicos ou catiônicos dissolvidos. Para isso utilizou-se o método da deriva de $\mathrm{pH}$ e de potencial Zeta, variando o intervalo de $\mathrm{pH}$ entre 2 e 11 mantendo constantemente as demais variáveis. Para a casca do arroz se obteve um PCZ de 5.40 e um PIE de 9.10 e para a casca de coco um PCZ de 4.61 e um PIE de 9.45, apontado uma distribuição de cargas predominantemente positiva sobre a sua superfície. A avaliação posterior desta característica comprovou sua finalidade para a remoção de corantes iônicos. Alcançou-se uma remoção de aproximadamente de 93\% do corante catiônico vermelho básico (VB46) e um $\mathrm{pH}$ maior que 5.0 e uma retenção total de aproximadamente $73 \%$ do corante aniônico vermelho 40 (V40) a um $\mathrm{pH}=2.0$ sobre a casca do arroz. A determinação do PCZ e do PIE dos absorventes possibilita o estabelecimento de melhores condições para a absorção de corantes iônicos na solução.

Palavras chave: Absorção, casca de arroz, casca de coco, vermelho básico 46, vermelho 40 .

\section{Introducción}

El vertiginoso crecimiento poblacional, y la subsecuente demanda de alimentos, han conducido a la generación y acumulación de enormes cantidades de residuos de tipo orgánico y sintético, derivados de su procesamiento, que representan un agravante adicional a la problemática de contaminación ambiental existente, debido a la generación de gases y lixiviados como resultado de su exposición a las condiciones climáticas y a la descomposición microbiana. Cuando estos gases y lixiviados migran al medio ambiente producen riesgos potenciales para la salud humana, así como la posibilidad de incendios, olores desagradables, contaminación del aire y calentamiento global (El-Fadel et al., 1997). 
Los graves y múltiples inconvenientes sociales y ambientales ocasionados por los residuos sólidos han llevado a la implementación de diversas leyes ambientales para su regulación, leyes que pueden ser retroactivas y obligaciones solidarias (Cheremisinoff, 2003). En particular, Colombia genera 10 millones de residuos sólidos al año, de los cuales el $77 \%$ son residuos domésticos y el $23 \%$ industriales. La magnitud registrada por estos desechos ha sido tal que afortunadamente se vienen desarrollando nuevas políticas encaminadas tanto a la gestión de su tratamiento como a la reutilización de los mismos en distintos sectores productivos. Estas políticas están contenidas principalmente en las leyes 99 de 1993 y 142 de 1994 de la Constitución Política Colombiana (Uribe \& Villa, 2010).

Como caso particular, cabe resaltar la utilización de residuos agroindustriales para el desarrollo de tecnologías alternativas de tratamiento de efluentes líquidos contaminados. Específicamente estos subproductos, han sido explorados como potenciales materiales adsorbentes para el proceso de adsorción, para el cual se han señalado ventajas notables como la remoción de la molécula completa del contaminante, alta eficiencia en la retención, bajo costo y facilidad de implementación del proceso, así como la ausencia de formación de lodos y/o productos tóxicos (Bhatnagar \& Minocha, 2006; Robinson et al., 2001; Ramakrishna \& Viraraghavan, 1997; Crini, 2006). Dicha fragmentación de las moléculas, o generación de sedimentos, son características muy usuales en las metodologías convencionales tales como la floculación, coagulación, sedimentación, tratamientos químicos de precipitación y métodos electroquímicos (Robinson et al., 2001; Banat et al., 1996; Forgacs et al., 2004).

La capacidad de adsorción de estos residuos agrícolas depende de múltiples factores, tales como el valor de $\mathrm{pH}$, dosificación, concentración del contaminante, tamaño de partícula, temperatura, tiempo de contacto y velocidad de agitación, es decir, de las condiciones bajo las cuales se lleva a cabo el proceso y de la naturaleza del contaminante (Hormaza, et a.,I 2012; Vijyakumar et al., 2012; El-Latif, et al., 2010; Wanchanthuek \& Thapol, 2011; Elkady et al., 2011). No obstante y de forma específica, la capacidad de adsorción del residuo está determinada por su composición química particular y por la distribución de cargas sobre su superficie (Menéndez et al., 1995; Appel et al., 2003). Así, la determinación del punto de carga cero y del punto isoeléctrico del adsorbente de interés permiten establecer dicha distribución de cargas global sobre su superficie y con ello su capacidad particular para retener contaminantes iónicos, tales como colorantes y metales. Así mismo, el punto de carga cero señala el intervalo más adecuado del valor de $\mathrm{pH}$ para alcanzar la remoción eficiente de un determinado contaminante de naturaleza aniónica o catiónica.

El punto de carga cero, PZC, se define como el valor del pH en el cual la carga neta total (externa e interna) de las partículas sobre la superficie del material adsorbente es neutra, es decir, el número de sitios positivos y negativos es igual (Franks \& Meagher, 2003); mientras que el punto isoeléctrico, IEP, se define como el valor correspondiente a las cargas superficiales externas del material. Estos parámetros son muy valiosos para determinar la afinidad particular de un determinado adsorbente por un adsorbato específico (Babic et al., 1999; Menéndez et al., 1995).

Algunas investigaciones se han enfocado a la determinación del PZC y IEP de diferentes minerales (Appel et al., 2003), hierro (Allende et al., 2007), fosfato de cobre (Sabás \& Romero, 2007), tierra fuller (Atun et al., 2003), hidroxiapatita y calcita (Gómez del Río et al., 2001) y carbón activado (Rivera-Utrilla et al., 2003), los cuales se han usado para la remoción de contaminantes en aguas, confirmando la importancia de estos parámetros en lo referente al tratamiento adecuado de efluentes líquidos. No obstante, la determinación de los parámetros PZC e IPE ha sido poco explorada en los residuos agroindustriales. 
En el caso de la remoción de colorantes, existen múltiples investigaciones, donde se ha evaluado una gran variedad de residuos agroindustriales, tales como celulosa (Annadurai et al., 2002), tallo de banano (Gupta et al., 2011), pulpa de remolacha (Aksu, et al., 2006), corteza de cacao (Theivarasu et al., 2011), tuza de maíz (Hormaza et al., 2012), residuos avícolas (Hormaza \& Suarez, 2009), bagazo de caña (Ashoka \& Inamdar, 2010), se han limitado a una exploración amplia del valor del pH (usualmente de 2 - 10) para encontrar la condición adecuada para este parámetro y con ello optimizar el proceso de remoción, ignorando que cada material tiene una distribución de carga neta sobre su superficie que facilita la retención de un determinado tipo de contaminantes.

En este sentido, un número limitado de trabajos describen la utilidad de la determinación del PZC como un apoyo en selección del valor propicio del $\mathrm{pH}$, al cual debe efectuarse el proceso de remoción del contaminante de estudio para alcanzar un proceso eficiente (Mohd-Salleh et al., 2011; Martin, 2008).

La cascarilla de arroz y la corteza de coco son residuos agrícolas de gran abundancia en la costa norte de Colombia, cuya capacidad adsorbente ha sido evaluada exitosamente en la remoción de efluentes contaminantes coloreados (Alemán, 2012).

Con el propósito de contribuir a la ratificación de la gran utilidad de la evaluación del PZC y IEP, en la presente investigación se determinaron estos parámetros para los residuos agrícolas cascarilla de arroz y corteza de coco, con el fin de conocer la distribución de cargas tanto en la superficie externa como interna de estos materiales, y de esta forma establecer la mejor relación adsorbenteadsorbato para un determinado proceso de remoción. Dichas características de carga de los adsorbentes fueron posteriormente evaluadas mediante la remoción de un colorante aniónico y catiónico.

\section{Materiales y métodos}

\section{Preparación del material adsorbente}

La cascarilla de arroz (CA) fue adquirida en el municipio de Montería, departamento de Córdoba. Este residuo se trituró en un molino de aspas, se lavó con agua destilada y con peróxido de hidrógeno $(0.5 \%)$. El material se sometió a secado durante 48 horas en un horno de convección forzada, luego se pasó por un tamiz de malla N. 35 para obtener un tamaño de partícula entre 0.30 y $0.50 \mathrm{~mm}$, con el cual se realizaron las mediciones.

La corteza de coco (CC) se recolectó en un mercado local de la ciudad de Medellín y se sometió a un pre-tratamiento que incluyó molienda, tamizado, lavado y secado. La reducción del tamaño del adsorbente se efectuó en un molino de aspas, seleccionando mediante tamizado un tamaño de partícula entre 0.5 y $0.7 \mathrm{~mm}$. El lavado se realizó repetidamente con agua destilada y con solución de peróxido de hidrógeno $(0.5 \%)$, finalmente el material fue secado en un horno de convección forzada a una temperatura de $80^{\circ} \mathrm{C}$ por un tiempo de 48 horas y almacenado herméticamente para la realización de las pruebas.

\section{Punto de carga cero (PZC)}

Se tomaron $50 \mathrm{~mL}$ de agua destilada en erlenmeyers de $100 \mathrm{~mL}$, ajustando el $\mathrm{pH}$ de cada solución entre 3.0 y 11 unidades, adicionando las cantidades adecuadas de $\mathrm{HCl} 0.1 \mathrm{M}$ e $\mathrm{NaOH} 0.1 \mathrm{M}$. A estas soluciones se añadió $0.5 \mathrm{~g}$ de muestra del material adsorbente y transcurridas 48 horas bajo agitación y a temperatura ambiente se procedió a medir el valor del $\mathrm{pH}$ final. EI PZC corresponde al punto donde la curva de $\mathrm{pH}$ final en función del $\mathrm{pH}$ inicial corta la diagonal.

Las mediciones del PZC y IEP se realizaron en un titulador automático Titrino plus 848 de marca Metrohm con electrodos de $\mathrm{pH}$ y de potencial. 


\section{Punto isoeléctrico IEP}

El IEP de cada adsorbente se determinó mediante medidas del potencial zeta. En erlenmeyers de 100 $\mathrm{mL}$ se adicionó $50 \mathrm{~mL}$ de agua destilada, ajustando el pH de cada solución entre 3.0 y 11 unidades, adicionando las cantidades adecuadas de $\mathrm{HCl} 0.1$ $\mathrm{M}$ e $\mathrm{NaOH} 0.1 \mathrm{M}$. A estas soluciones se agregó $0.5 \mathrm{~g}$ de muestra del adsorbente y se sometió a agitación por 2 horas. Transcurrido este tiempo se midió el potencial zeta usando el pH-metro con el electrodo de potencial. El punto isoeléctrico, IEP, corresponde al valor del $\mathrm{pH}$ en el cual el potencial zeta es cero.

\section{Estudio de adsorción}

Los ensayos de adsorción para los dos colorantes fueron realizados por triplicado y bajo sistema discontinuo, midiendo la absorbancia final del contaminante en un espectrofotómetro UV-Vis de doble haz, marca Perkin Elmer Lamba - 35. Las variables, dosificación, tamaño de partícula, tiempo de contacto, velocidad de agitación, temperatura y concentración inicial de colorante fueron mantenidas constantes para el colorante aniónico y catiónico evaluado.

\section{Colorante aniónico Rojo 40 (R40)}

Se realizó una curva de calibración del colorante $\mathrm{R} 40$ entre $0.0 \mathrm{mg} / \mathrm{L}$ y $60 \mathrm{mg} / \mathrm{L}$ en erlenmeyers de $100 \mathrm{~mL}$ con $1.0 \mathrm{~g}$ de cascarilla de arroz, cuyo tamaño de partícula osciló entre $0.3-0.5 \mathrm{~mm}$, se adicionó solución acuosa con colorante R40 de 30 $\mathrm{mg} / \mathrm{L}$, y se ajustó el $\mathrm{pH}$ de cada solución en un intervalo de 2.0 - 6.0 con adiciones sucesivas de $\mathrm{NaOH}$ y $\mathrm{HCl} 0.1 \mathrm{M}$ y se sometió a agitación durante 24 horas a $160 \mathrm{rpm}$ a temperatura ambiente. Al cabo de este tiempo se midió la concentración de la solución en equilibrio para determinar el porcentaje de remoción del colorante, mediante la ecuación 1.
$\%$ Remoción $=\frac{\text { Co-Ceq }}{\text { Co }} \times 100 \%$

Donde Co: Concentración inicial (mg/L)

Ceq: Concentración en equilibrio (mg/L)

\section{Colorante catiónico Rojo básico 46 (RB46)}

Para el RB46 se efectuó una curva de calibración entre $0.0 \mathrm{mg} / \mathrm{L}$ y $60 \mathrm{mg} / \mathrm{L}$. En erlenmeyers de 100 $\mathrm{mL}$ con $1.0 \mathrm{~g}$ de cascarilla de arroz con tamaño de partícula entre $0.3-0.5 \mathrm{~mm}$, se adicionó solución acuosa con RB46 de $30 \mathrm{mg} / \mathrm{L}$, ajustando el $\mathrm{pH}$ de cada erlenmeyer en un intervalo de 2.0 - 10.0 con adiciones sucesivas de $\mathrm{NaOH}$ y $\mathrm{HCl} 0.1 \mathrm{M}$ y se sometió a agitación durante 24 horas a 160 rpm a temperatura ambiente. Al cabo de este tiempo se procedió a medir la concentración de la solución en equilibrio para determinar el porcentaje de remoción del colorante, mediante la ecuación 1.

\section{Resultados y discusión}

\section{Determinación del PZC y IEP}

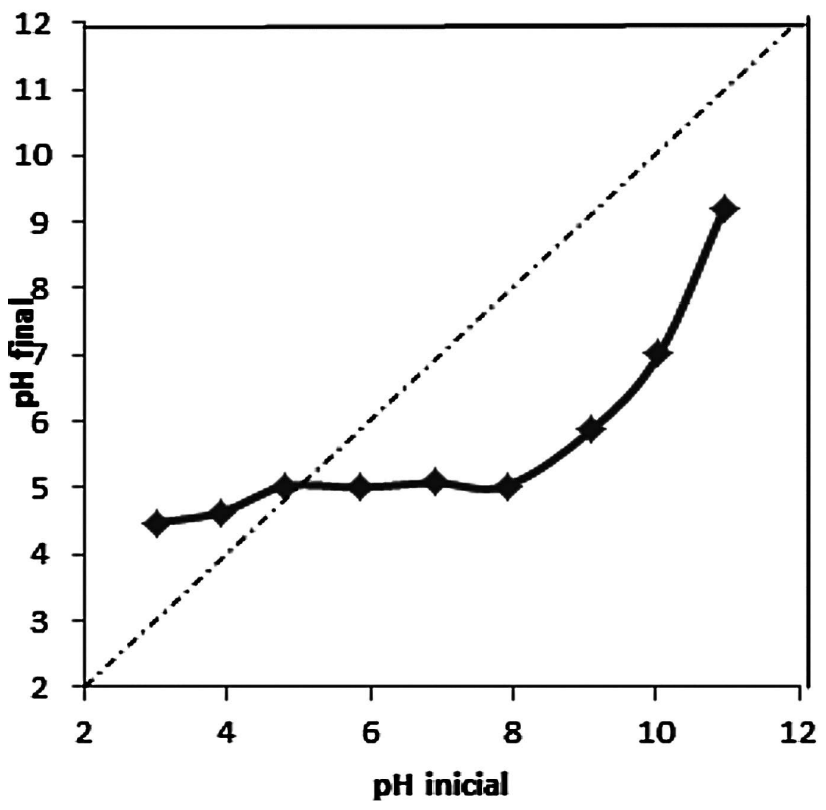

Figura 1. $P Z C$ de la $C A$ 


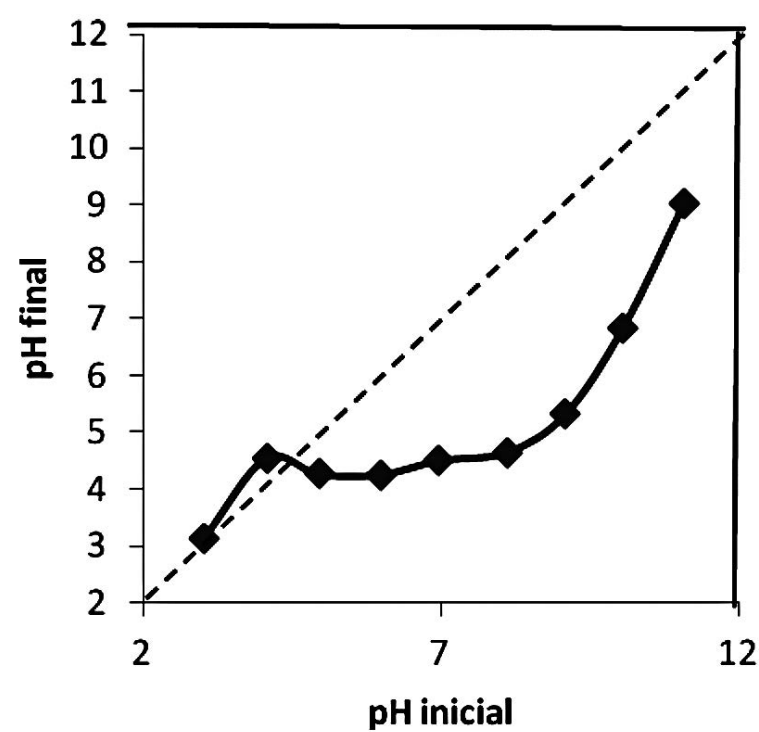

Figura 2. IEP de la CA

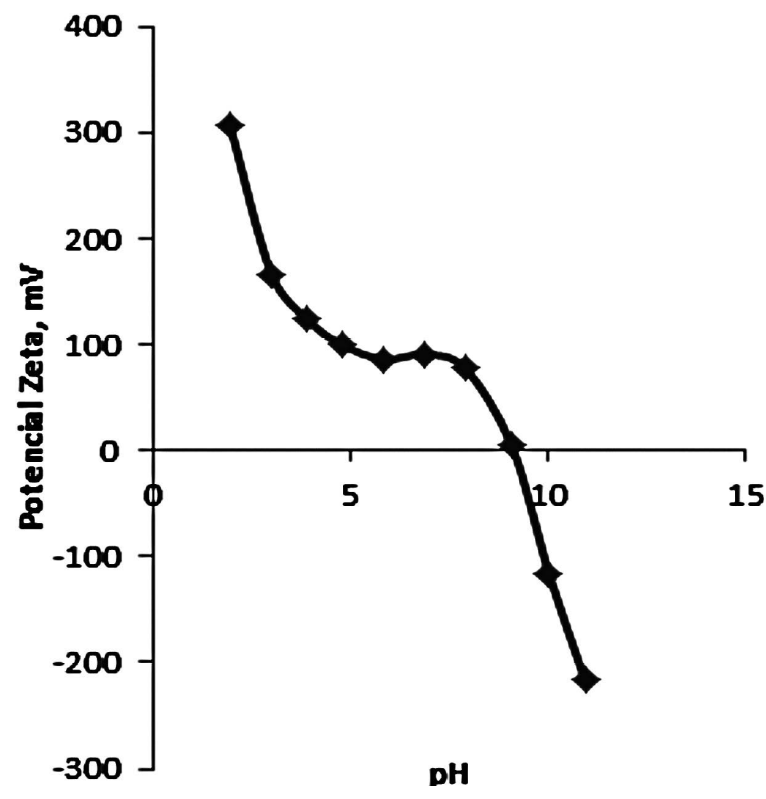

Figura 3. PZC de la CC

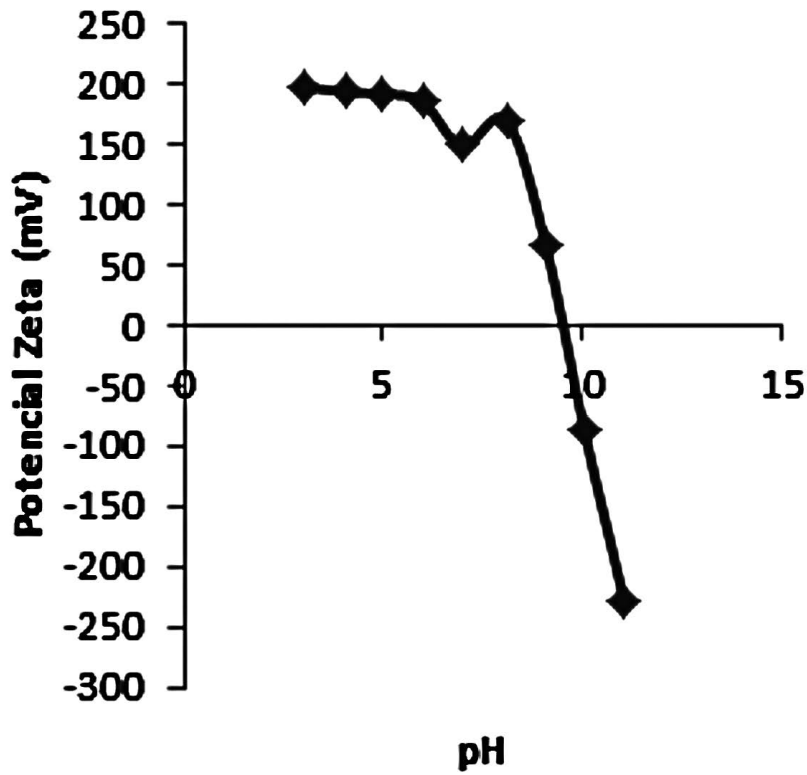

Figura 4. IEP de la CA

EI PZC fue determinado por el método de la deriva del $\mathrm{pH}$, evaluando un intervalo de $\mathrm{pH}$ entre $3.0 \mathrm{y}$ 11. Los valores de PZC encontrados son 5.40 y 4.61 para CA y CC respectivamente (Tabla 1).

Tabla 1. PZC Y IEP de la CA y CC

\begin{tabular}{lcc}
\hline Material & $\begin{array}{c}\text { Punto de carga } \\
\text { cero (PZC) }\end{array}$ & $\begin{array}{c}\text { Punto } \\
\text { Isoeléctrico } \\
\text { (IEP) }\end{array}$ \\
\hline $\begin{array}{l}\text { Cascarilla } \\
\text { de arroz }\end{array}$ & 5.40 & 9.10 \\
$\begin{array}{l}\text { Corteza } \\
\text { de coco }\end{array}$ & 4.61 & 9.45 \\
\hline
\end{tabular}


Los valores similares de PZC obtenidos para estos dos residuos agroindustriales son evidentes al comparar la Figura 1 y 3 , donde se aprecia la similitud de estos dos materiales absorbentes entre un valor de $\mathrm{pH}$ de 4.5 y 11 . Su diferencia radica específicamente en la zona de $\mathrm{pH}$ entre $3.0-4.5$ señalando un acelerado aumento a $\mathrm{pH} 4.0$ para $\mathrm{CC}$, en tanto que el aumento en $\mathrm{CA}$ es progresivo en este mismo valor de $\mathrm{pH}$.

El IEP fue determinado por el método del potencial zeta para los dos adsorbentes de estudio, considerando un intervalo de $\mathrm{pH}$ entre 2.0 y 11, encontrando valores de 9.10 y 9.45 para CA y CC correspondientemente (Tabla 1). A pesar de la similitud de los valores del IEP para estos dos materiales, su gráfica de potencial Vs valor de $\mathrm{pH}$ describe notables diferencias. Así para CA se observa una disminución progresiva en el valor del potencial a medida que aumenta el valor del pH (3.0-7.0), en tanto que para CC esta disminución solo inicia desde un valor de $\mathrm{pH}=6.5$. La diferencia encontrada tanto en el PZC como del IEP en estos dos materiales puede ser explicada debido a su diferente composición lignocelulósica. En particular el contenido de celulosa y hemicelulosa difiere notablemente, con un porcentaje de $60.17 \%$ y $14.82 \%$ respectivamente para la CA (Alemán, 2012) y un $77.6 \%$ y $13.4 \%$ para la CC.

\section{Análisis de la remoción de colorantes}

Dado que el PZC corresponde a un punto de equilibro de cargas sobre el material absorbente, valores de $\mathrm{pH}$ mayores que $\mathrm{PZC}$ generan una superficie cargada negativamente, en tanto que un $\mathrm{pH}$ menor que PZC una superficie cargada positivamente. De allí que la determinación de este parámetro sea de gran ayuda para establecer las condiciones propicias en cuanto al valor de $\mathrm{pH}$ que permite alcanzar una remoción eficiente de un determinado colorante. Para el caso de la CA se prevé que colorantes de naturaleza aniónica serán retenidos a un $\mathrm{pH}$ inferior a 5.4 y que la remoción de los colorantes catiónicos será favorecida a pH superiores a este valor de PZC.

Con el propósito de verificar este comportamiento, es decir, la presencia de una superficie cargada positiva o negativamente sobre $\mathrm{CA}$, se llevaron a cabo ensayos de adsorción bajo sistema discontinuo, manteniendo como fijas las variables dosificación, tamaño de partícula, tiempo de contacto, velocidad de agitación, temperatura y concentración del colorante catiónico y aniónico.

La adsorción del colorante aniónico rojo 40, (R40), es mostrada en la Figura 5, donde se aprecia que una máxima remoción del orden del $73 \%$ fue alcanzada para este colorante en medio ácido $(\mathrm{pH}$ = 2.0). Este porcentaje disminuye drásticamente a medida que se incrementa el valor del $\mathrm{pH}$, reduciéndose a un $25 \%$ a un $\mathrm{pH}=3.0$ y llegando una remoción mínima del $10 \%$ a $\mathrm{pH}=5.0$, valor cercano al PZC de este adsorbente.

Por otro lado y de acuerdo a lo previsto, se aprecia que la remoción del colorante catiónico rojo básico 46, (RB46), se favoreció a pH superiores al valor del PZC (PZC = 5.4) como resultado de la generación de cargas positivas sobre la superficie del adsorbente, (Figura 6). En este caso y a diferencia de lo observado para el colorante aniónico $\mathrm{R} 40$, un $\mathrm{pH}$ ácido, $(\mathrm{pH}=2.0)$, conduce al mínimo porcentaje de remoción del RB46 sobre el mismo adsorbente, (adsorción del 81\%). La retención de RB46 incrementa notablemente a partir de valores de $\mathrm{pH}$ superiores a 5.0 , obteniendo un porcentaje del orden del 93\% a un $\mathrm{pH}=6.5$. Un incremento posterior en el valor del $\mathrm{pH}$ no conduce a un mayor porcentaje de remoción, hecho que puede ser explicado como consecuencia de la saturación de sitios cargados positivamente sobre la superficie del adsorbente. 
La remoción del $74 \%$ obtenida para el colorante aniónico R40 puede ser mejorada mediante la variación simultánea de los parámetros del proceso a través de un diseño estadístico de experimento.

Finalmente, la diferencia en la remoción obtenida de los colorantes RB46 y R40 sobre CA, bajo iguales condiciones de operación, permite sugerir que su superficie es más adecuada para la retención de colorantes de naturaleza catiónica.

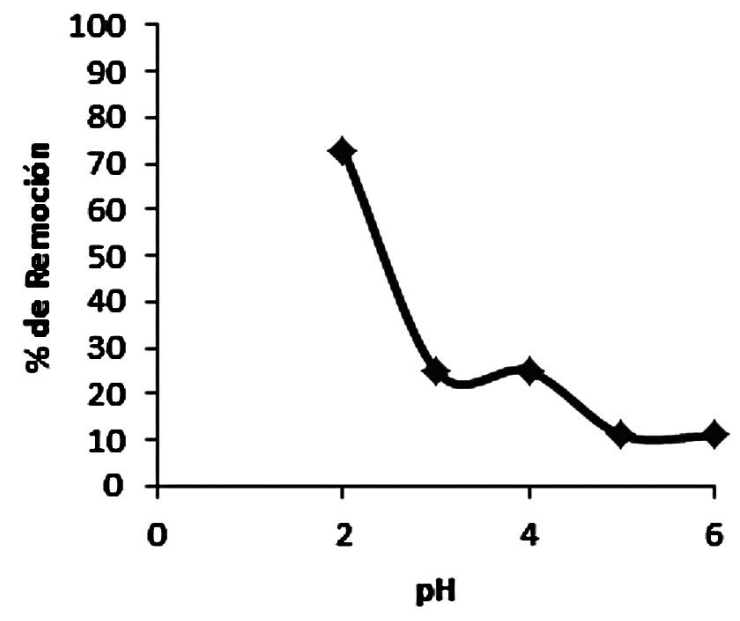

Figura 5. Adsorción de colorante aniónico R40. Ci R40: $30 \mathrm{mg} / \mathrm{L}$; Tamaño de partícula: 0.3-0.5 mm; dosificación de CA: $1.0 \mathrm{~g}$; Tiempo de contacto: 24 h; Velocidad de agitación: 160 rpm; Temperatura: $25 \pm 2^{\circ} \mathrm{C}$.

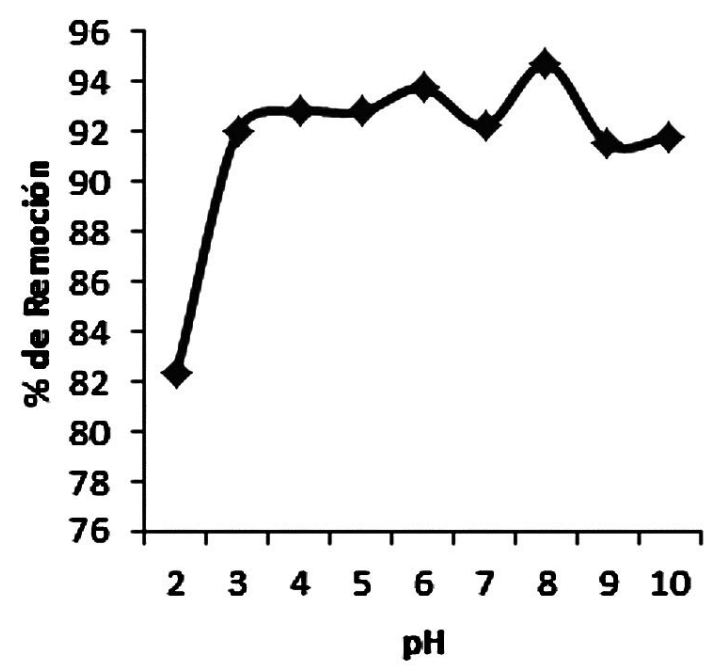

Figura 6. Adsorción de colorante catiónico RB46. Ci RB46: 30 mg/L; Tamaño de partícula : 0.3-0.5 $\mathrm{mm}$; Dosificación de CA: $1.0 \mathrm{~g}$; Tiempo de contacto: $24 \mathrm{~h}$; Velocidad de agitación : $160 \mathrm{rpm}$; Temperatura: $25 \pm 2^{\circ} \mathrm{C}$.
Resultados similares, que confirman la utilidad del manejo del pH a partir del valor del PZC, son previstos para la $\mathrm{CC}$; a la fecha se evalúa la remoción del colorante aniónico R40 y catiónico RB46 sobre este adsorbente, cuyos resultados y optimización del proceso serán divulgados en un próximo artículo.

\section{Conclusiones}

Pocos estudios sobre adsorción se han interesado en la evaluación del PZC, no obstante este parámetro es realmente valioso en la selección del mejor intervalo de $\mathrm{pH}$ al que se debería llevar a cabo la remoción de un determinado contaminante, permitiendo reducir costos de utilización de equipos y horas de trabajo.

La deriva del $\mathrm{pH}$ constituye un método alternativo, económico y rápido para la determinación del PZC de adsorbentes, como los residuos agrícolas, y con ello poder establecer la distribución de cargas de su superficie externa e interna. De igual forma, El potencial zeta representa un método adecuado para determinar el IEP de materiales adsorbentes.

El valor de PZC obtenido en la presente investigación para CA y CC fue de 5.40 y 4.61 respectivamente. A partir de este valor, fue posible remover de manera eficiente el colorante catiónico RB46 y el colorante aniónico R40 sobre CA en un intervalo seleccionado de $\mathrm{pH}$.

\section{Agradecimientos}

Los autores expresan agradecimientos a la Universidad Nacional de Colombia, Vicerrectoría de Investigación - Dirección Nacional de Extensión por la financiación del proyecto Código 400000011002 en la Convocatoria Apoyo a Proyectos y Actividades de Extensión en la modalidad Innovación y Gestión Tecnológica, GTI, Año 2012. Así mismo, a la Dirección de Investigación de la Sede Medellín, DIME, por el respaldo económico a través de la Convocatoria de Fortalecimiento de la formación para la Investigación, Año 2013. Proyecto Código 18484. 


\section{Literatura citada}

1. Aksu, Z. \& Isoglu, A. (2006). Use of agricultural waste sugar beet pulp for the removal of Gemazol turquoise blue-G reactive dye from aqueous solution. Journal of Hazardous Materials. 137 (1): 418-430.

2. Alemán, A. (2012). Evaluación de la esterificación sobre cascarilla de arroz como estrategia para incrementar la capacidad de remoción del colorante rojo básico 46. Tesis de Maestría en Ciencias Químicas. Medellín: Facultad de Ciencias-Escuela de Química Medellín Universidad Nacional de Colombia.

3. Allende, M., Romero, E. \& Reyez, L. (2007). Caracterización de compuestos de hierro, como material reactivo para inmovilizar $\mathrm{Cr}(\mathrm{VI})$ en suelo contaminado. Memorias en extenso. VI Congreso Internacional y XII nacional de Ciencias Ambientales. 1-6. Chihuahua: Universidad Autónoma del Estado de México.

4. Annadurai, G., Juang, R. \& Lee, D. (2002). Use of cellulose-based wastes for adsorption of dyes from aqueous solutions. Journal of Hazardous Materials. 92 (3): 263-274.

5. Appel, C. Ma, L., Dean, R. \& Kennelly, E. (2003). Point of zero charge determination in soils and minerals via traditional methods and detection of electroacoustic mobility. Geoderma. 113 (1-2): 77-93.

6. Ashoka, H. \& Inamdar, S. (2010). Adsorption Removal of methyl red from aqueous solutions with treated sugarcane bagasse and activated carbón- a comparative study. Global Journal of Enviromental Research. 4(3): 175-182.

7. Atun, G., Hisarli, G., Sheldrick, W. \& Muhler, M. (2003). Adsorptive removal of methylene blue from colored effluents on fuller's earth. Journal of Colloid and Interface Science. 261 (1): 32-39.

8. Babic, B., Milonjic, S., Polovina, M. \& Kaludierovic, B. (1999). Point of zero charge and intrinsic equilibrium constants of activated carbon cloth. Carbon. 37 (3): 477-481.

9. Banat, I., Nigam, P., Singh, D. \& Marchant, R. (1996). Microbial decolorization of textile-dyecontaining effluents: A review. Bioresource Technology. 58 (3): 217- 227.

10. Bhatnagar, T. \& Minocha, A. (2006). Conventional and non-conventional adsorbents for removal of pollutants from water: A review. Indian Journal of Chemical Technology. 13: 203-217.

11. Cheremisinoff, N. (2003). Environmental Laws and Regulatory Drivers. (Chap. 2) 23-33. Handbook of Solid Waste Management and Waste Minimization Technology. Burlington: Elsevier.

12. Crini, G. (2006). Non-conventional low-cost adsorbents for dye removal: A review. Bioresource Technology. 97 (9): 1061-1085.

13. El-Fadel, M., Findikakis, A. \& Leckie, J. (1997) Environmental Impacts of Solid Waste Landfilling. Journal of Environmental Management. 50 (1): 1-25.
14. El-Latif, A., Ibrahimz, A. \& El-Kady. (2010). Adsorption Equilibrium, kinetics and thermodynamics of methylene blue from aqueous solutions using biopolymer oak sawdust composite. Journal of American Science. 6 (6): 267-283.

15. Elkady, M., Ibrahim, A. \& El-Latif, A. (2011). Assessment of the adsorption kinetics, equilibrium and thermodynamic for the potential removal of reactive red dye using eggshell biocomposite beads. Desalination. 278 (1-3): 412-423.

16. Forgacs, E., Cserháti, T. \& Oros, G. (2004). Removal of synthetic dyes from wastewaters: a review. Environment International. 30 (7): 953- 971.

17. Franks, G. \& Meagher, L. (2003). The isoelectric points of sapphire crystals and alpha-alumina powder. Colloids and Surfaces A: Physicochem. Eng. Aspects. 214 (1-3): $99-/ 110$

18. Gómez del Río, J., Cicerone, D. \& Morando, P. (2001). Aplicación de materiales naturales a purificación de efluentes: columnas de hidroxiapatita y calcita como intercambiadores de metales pesados. 25-32. Misiones: Jornadas SAM - CONAMET - AAS 2001.

19. Gupta, N., Kushwaha, A. \& Chattopadhyaya, M. (2011). Kinetics and thermodynamics of malachite green adsorption on banana pseudo-stem fibers. Journal of Chemical and Pharmaceutical Research. 3(1): 284-296.

20. Hormaza, A., Figueroa, D. \& Moreno, A. (2012). Evaluación de la remoción de un colorante azo sobre tuza de maíz mediante diseño estadístico. Revista de la Facultad de Ciencias. 1 (1): 61-71.

21. Hormaza, A. \& Suarez, E. (2009). Estudio del proceso de biosorción de dos colorantes estructuralmente diferentes sobre residuos avícolas. Rev. Soc. Quím. Perú. 75 (3): 329-338.

22. Martin, M. (2008). Caracterización y aplicación de biomasa residual a la eliminación de metales pesados. Tesis Doctoral, Departamento de Ingeniería Química. Granada: Universidad de Granada.

23. Menéndez, J., Illán -Gómez, C. \& Radovic, R. (1995). On the difference between the isoelectric point and the point of zero charge of carbons. Carbon. 33 (11): 1655-1659.

24. Mohd Salleh, M., Mahmoud, D., Abdul Karim, W. \& Idris, A. (2011). Cationic and anionic dye adsorption by agricultural solid wastes: A comprehensive review. Desalination, 280 (1-3): 1-13.

25. Rivera-Utrilla, J., Bautista-Toledo, I., Ferro-Garcia, M. \& Moreno-Castilla, C. (2003). Bioadsorption of $\mathrm{Pb}(\mathrm{II})$, $\mathrm{Cd}(\mathrm{II})$, and $\mathrm{Cr}(\mathrm{VI})$ on activated carbon from aqueous solutions. Carbon. 41: 323-330.

26. Ramakrishna, R. \& Viraraghavan, T. (1997). Dye removal using low cost adsorbents. Water Sci. Technol. 36: 189-196 
27. Robinson, T., McMullan, G., Marchant, R. \& Nigam, P. (2001). Remediation of dyes in textile effluent: a critical review on current treatment technologies with a proposed alternative. Bioresource Technology. 77 (3): 247-255.

28. Sabás, L. \& Romero, E. Síntesis y caracterización del $\mathrm{Cu}_{3}\left(\mathrm{PO}_{4}\right)_{2}$ para eliminar contaminantes del agua. Memorias en Extenso. 7-10. VI Congreso Internacional y XII Nacional de Ciencias Ambientales. Chihuahua: Universidad Autónoma del Estado de México.

29. Theivarasu, C., Mylsamy, S. \& Sivakumar, N. (2011). Cocoa Shell as Adsorbent for the Removal of Methylene Blue from Aqueous Solution: Kinetic and Equilibrium Study. Universal Journal of Environmental Research and Technology. 1: 70-78.
30. Vijyakumar, G., Tamilasaran, R. \& Dharmendirakumar, M. (2012). Adsorption, Kinetic, Equilibrium and Thermodynamic studies on the removal of basic dye Rhodamine- $B$ from aqueous solution by the use of natural adsorbent perlite. Journal of Materials of Enviromental Sciences 3 (1): 157-170.

31. Uribe, L. \& Villa, M. (2010). Colombia (Chapter 10). In: The International Comparative Legal Guide to: Environment Law 2010. A practical croos-border insight into environment law. 76-83, London: Ed. Global Legal Group Ltd.

32. Wanchanthuek, R. \& Thapol, A. (2011). The Kinetic study of methylene blue adsorption over MgO from PVA template preparation. Journal of Enviromental Science and Technology. 4 (5): 552-559. 